PAPER • OPEN ACCESS

Out-of-field doses for scanning proton radiotherapy of shallowly located paediatric tumours - a comparison of range shifter and 3D printed compensator

To cite this article: A Wochnik et al 2021 Phys. Med. Biol. 66035012

View the article online for updates and enhancements.

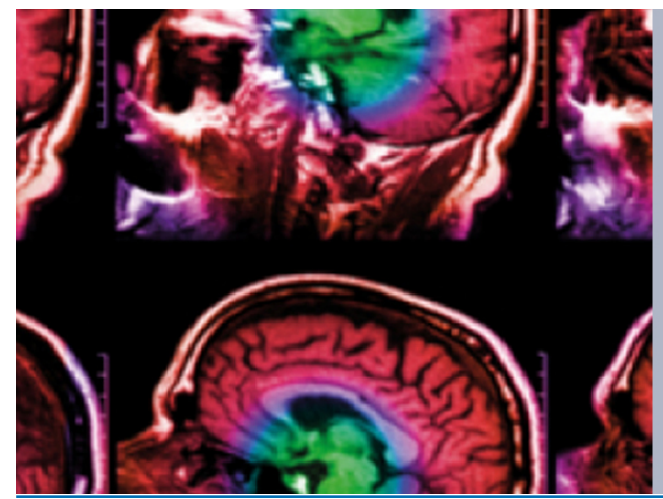




\section{Out-of-field doses for scanning proton radiotherapy of shallowly located paediatric tumours-a comparison of range shifter and 3D printed compensator}

RECEIVED

6 July 2020

REVISED

23 October 2020

ACCEPTED FOR PUBLICATION

17 November 2020

PUBLISHED

25 January 2021

Original content from this work may be used under the terms of the Creative Commons Attribution 4.0 licence.

Any further distribution of this work must maintain attribution to the author(s) and the title of the work, journal citation and DOI.

\author{
A Wochnik ${ }^{1}$ (D) , L Stolarczyk ${ }^{1,2,3}$ (D) I Ambrožová ${ }^{4}$, M Davídková $^{4}$, M De Saint-Hubert ${ }^{5}$, Somański $^{6}$ (D),

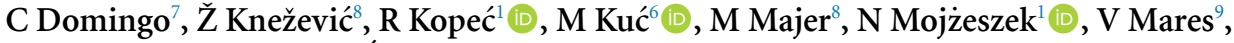 \\ I Martínez-Rovira $^{7}$ (D) M Á Caballero-Pacheco ${ }^{7}$ (D) E Pyszka $^{1}$, J Swakońn ${ }^{1}$ D , S Trinkl ${ }^{9,10} \mathbb{D}$, M Tisi $^{9}$, \\ R Harrison ${ }^{11}$ and P Olko ${ }^{1}$ \\ 1 Institute of Nuclear Physics PAN, Radzikowskiego 152, Krakow 31-342, Poland \\ 2 Skandionkliniken, von Kraemers Allé 26, Uppsala 752 37, Sweden \\ 3 Dansk Center for Partikelterapi, Palle Juul-Jensens Boulevard 25, 8200 Aarhus N, Denmark \\ 4 Department of Radiation Dosimetry, Nuclear Physics Institute Czech Academy of Sciences, Prague CZ-250 68 Řež, Czech Republic \\ 5 Belgium Nuclear Research Centre (SCK CEN), Boeretang 200, Mol BE-2400, Belgium \\ 6 National Centre for Nuclear Research, Otwock-Świerk 05-400, Poland \\ 7 Departament de Física, Universitat Autònoma de Barcelona (UAB), Bellaterra E-08193, Spain \\ 8 Ruđer Bošković Institute, Bijenička c. 54, Zagreb 10000, Croatia \\ 9 Helmholtz Zentrum München, Institute of Radiation Medicine, Ingolstädter Landstraße 1, Neuherberg 85764, Germany \\ 10 Technische Universität München, Physik-Department, Garching 85748, Germany \\ 11 University of Newcastle upon Tyne, Tyne and Wear, Newcastle upon Tyne NE1 7RU, United Kingdom \\ E-mail: Agnieszka.Wochnik@ifj.edu.pl
}

Keywords: scanning proton radiotherapy, measurement of stray neutrons, ambient dose equivalent, active detectors, passive detectors, anthropomorphic paediatric phantom measurements, secondary radiation measurements

Supplementary material for this article is available online

\section{Abstract}

The lowest possible energy of proton scanning beam in cyclotron proton therapy facilities is typically between 60 and $100 \mathrm{MeV}$. Treatment of superficial lesions requires a pre-absorber to deliver doses to shallower volumes. In most of the cases a range shifter (RS) is used, but as an alternative solution, a patient-specific 3D printed proton beam compensator (BC) can be applied. A BC enables further reduction of the air gap and consequently reduction of beam scattering. Such pre-absorbers are additional sources of secondary radiation. The aim of this work was the comparison of RS and BC with respect to out-of-field doses for a simulated treatment of superficial paediatric brain tumours. EURADOS WG9 performed comparative measurements of scattered radiation in the Proteus C-235 IBA facility (Cyclotron Centre Bronowice at the Institute of Nuclear Physics, CCB IFJ PAN, Kraków, Poland) using two anthropomorphic phantoms- 5 and $10 \mathrm{yr}$ old-for a superficial target in the brain. Both active detectors located inside the therapy room, and passive detectors placed inside the phantoms were used. Measurements were supplemented by Monte Carlo simulation of the radiation transport. For the applied 3D printed pre-absorbers, out-of-field doses from both secondary photons and neutrons were lower than for RS.

Measurements with active environmental dosimeters at five positions inside the therapy room indicated that the $\mathrm{RS} / \mathrm{BC}$ ratio of the out-of-field dose was also higher than one, with a maximum of 1.7. Photon dose inside phantoms leads to higher out-of-field doses for RS than BC to almost all organs with the highest RS/BC ratio 12.5 and 13.2 for breasts for 5 and $10 \mathrm{yr}$ old phantoms, respectively. For organs closest to the isocentre such as the thyroid, neutron doses were lower for $\mathrm{BC}$ than RS due to neutrons moderation in the target volume, but for more distant organs like bladder-conversely-lower doses for $\mathrm{RS}$ than $\mathrm{BC}$ were observed. The use of $3 \mathrm{D}$ printed $\mathrm{BC}$ as the pre-absorber placed in the near vicinity of patient in the treatment of superficial tumours does not result in the increase of secondary radiation compared to the treatment with RS, placed far from the patient. 


\section{Introduction}

Proton therapy (PT) is a modern method of radiotherapy using proton beams of energies up to $250 \mathrm{MeV}$, in which a steady increase in the number of patients and emerging centres offering this type of treatment is observed. One of the main arguments for the application of PT is favourable dose distributions, because protons deposit maximum energy at the end of their path. That feature allows the achievement of improved precision of the dose delivery while reducing the risk of damage to critical organs and healthy tissue surrounding the lesion (Tian et al 2018). Out-of-field doses from scattered radiation absorbed by normal tissues increase the probability of late effects including the generation of secondary cancers, particularly in children (Packer et al 2013). Neutron doses are of special concern because of their high biological effectiveness (Valentin 2003).

Undesirable doses to healthy tissues distant from the target volume largely depend on the beam delivery technique. In PT facilities with double beam scattering techniques, a significant secondary neutron component is generated in the beam forming elements (Stolarczyk et al 2011, Bonfrate et al 2016). This disadvantage has been considerably reduced with the introduction of pencil beam scanning (PBS) technology. The beam is steered by magnets and only thin beam monitoring chambers and a vacuum foil intercept the proton trajectories. Currently, practically all newly installed PT facilities are equipped with the PBS system. In cyclotrons, the proton beam is extracted at the maximal energy of $230 \mathrm{MeV}$ (IBA, Sumitomo) or $250 \mathrm{MeV}$ (Varian) and then the beam energy is reduced by an energy degrader and energy selector to adapt the beam energy to the required range.

In PT of children, compared with adults, special attention is given to the treatment of shallowly-located tumours. For young children, lesions might be located closer than a few centimetres beneath the patient's skin. In facilities with PBS technology, the lowest energy of the proton beam ranges typically between 60 and $100 \mathrm{MeV}$, which corresponds to a range of about 3.1-7.5 cm in water-equivalent thickness. The irradiation of superficial lesions requires the application of a pre-absorber, which reduces proton range. Typically a plate of poly(methyl methacrylate) (PMMA) or Lexan acting as a range shifter (RS) is attached to the nozzle at a certain distance from the patient's body and used to deliver shallower spots (Gillin et al 2010, Matysiak et al 2016). Increasing the distance between the RS and the patient's body increases the spot size and penumbra (Titt et al 2010, Shen et al 2015, Baumer et al 2018). To mitigate this effect, some PBS nozzles offer the possibility of minimizing the air gap by using a movable snout extension and bringing the RS closer to the patient (Pedroni et al 2005, Shen et al 2015, Matysiak et al 2016). However, the fixed shape of the RS plate makes it impossible to completely eliminate the air gap and requires special care to avoid collision with the patient. For nozzles without a movable RS, treatment of shallow tumours with high precision is significantly more difficult.

One of the solutions suggested in the literature is the use of a pre-absorber that will adhere exactly to the patient's body. Such a compensator should be designed and manufactured for each individual patient. Moreover, the thickness and final shape could also be adapted to the volume of irradiated lesion. An individual approach is possible using the additive technology of $3 \mathrm{D}$ printers for the production of compensators. In radiotherapy, several uses of 3D print-outs have been reported in the literature, as boluses in conventional therapy (Burleson et al 2015, Zou et al 2015, Canters et al 2016, tukowiak et al 2016, Park et al 2016, Oh et al 2017) and compensators in passively scattered beam PT (Ju et al 2014). Michiels et al (2018) presented the benefits of using the compensator relative to the RS for PBS technology using Monte Carlo (MC) simulations.

The question arises if the application of the compensator will influence patient exposure to secondary radiation compared with conventionally applied RSs. EURADOS Working Group 9 (Radiation Dosimetry in Radiotherapy) in previous years has focused on the determination of scattered radiation doses generated during treatment with modern radiotherapy techniques, using both photon and proton beams (Farah et al 2015, Mojżeszek et al 2017, Stolarczyk et al 2018). Particular attention was paid to paediatric patients treated with protons, e.g. out-of-field doses in critical organs inside paediatric phantoms in Knežević et al (2018).

In this work, comprehensive measurements of out-of-field neutron and photon doses produced during the treatment with PBS, were performed by EURADOS WG9. The measurements aimed at comparing the scattered radiation doses for two types of pre-absorbers-a $4 \mathrm{~cm}$ thick RS made of Lexan (polycarbonate resin thermoplastic), installed at the gantry scanning nozzle, as well as a $3 \mathrm{D}$ printed proton beam compensator (BC) with a uniform thickness of $4 \mathrm{~cm}$ attached directly to the patient's mask. The uniform density of the printed BC was confirmed by the CT scan. The doses from secondary radiation received by critical organs during irradiation of a superficial brain tumour, as well as the doses around the phantoms were measured. Inside the phantoms, doses were determined with the same set of passive solid state detectors as used in previous WG9 experiments: luminescent, track-etched and bubble detectors. Scattered radiation in the therapy room was measured using different neutron monitors. 


\section{Materials and methods}

\subsection{Measurements conditions and prescribed doses}

Measurements were performed at the Cyclotron Centre Bronowice (CCB IFJ PAN, Krakow) equipped with an Ion Beam Application gantry (IBA Proton Therapy System-Proteus 235) with a dedicated scanning nozzle. Experiments were carried out using two phantoms (see section 2.2)—corresponding to 5 and $10 \mathrm{yr}$ old children. For each phantom, two treatment plans were prepared using the Varian Eclipse treatment planning system (version 13.6) with two types of pre-absorbers, RS and BC. As a target, a $6 \mathrm{~cm}$ diameter sphere, shallowly located on the left-anterior side of the head with the isocentre in the middle of slice 3 (as shown in figure 1 the supplementary data) was chosen, similar to that described in Majer et al (2017), but with the target shifted towards the surface. The energies of layers were in the range from 80 to $140 \mathrm{MeV}$ for both phantoms. The spot size of beams, in terms of their sigma ${ }^{12}$ value in air, measured at the distance of $46 \mathrm{~cm}$ from the nozzle surface (at the isocentre), ranged between 5.3 and $4.4 \mathrm{~mm}$ at beam energies 100 and $140 \mathrm{MeV}$, respectively. To obtain a uniform dose distribution in the target, two irradiation fields at gantry angles of $0^{\circ}$ (F1) and $90^{\circ}$ (F2) were used. For both fields the source-to-surface distances were similar: 193.6 (F1), 194.6 (F2) and 193.7 (F1), $194.7 \mathrm{~mm}$ (F2) for RS and BC plans, respectively. The planned physical doses to the target were: $D_{\mathrm{T}}=100 \mathrm{~Gy}$ for irradiation of luminescent dosimeters, $D_{\mathrm{T}}=40$ Gy for track-etched detectors and $D_{\mathrm{T}}=2 \mathrm{~Gy}$ for bubble detectors (BD), adapted to the sensitivity of detectors in the positions of measurements. The doses correspond to $D_{\mathrm{RBE}}=110 \mathrm{~Gy}(\mathrm{RBE}), D_{\mathrm{RBE}}=44 \mathrm{~Gy}(\mathrm{RBE})$ and

$D_{\mathrm{RBE}}=2.2 \mathrm{~Gy}(\mathrm{RBE})$, respectively. Reference proton beam dosimetry was performed with a Marcus type ionisation chamber (PTW) connected to a Unidos Webline electrometer (PTW).

\subsection{Anthropomorphic paediatric phantoms}

Anthropomorphic phantoms representing 5 and $10 \mathrm{yr}$ old children (type 705D and type 706D, respectively) were used for this study (ATOM, Computerized Imaging Reference Systems, Inc. Norfolk, VA, USA). The phantoms consist of 26 and 32 slices for 5 and $10 \mathrm{yr}$ old phantoms, respectively, with each slice $25 \mathrm{~mm}$ thick. Slices are made of tissue equivalent material and divided into areas corresponding to individual organs, where detectors can be inserted into holes of diameter $5 \mathrm{~mm}$ and fixed with appropriate plugs. There are 180 and 213 detector holes in the 5 and $10 \mathrm{yr}$ old phantom, respectively. Based on phantom computed tomography, the distance between all detectors and the isocentre located in the middle of the spherical target was calculated. Mean organ doses in phantoms were estimated as the average dose measured by all passive detectors placed in the particular organs. The brain doses were scored separately in the target volume (defined as 'Brain target') to those outside ('Brain non target'). Measured doses were normalized to the physical target dose $D_{\mathrm{T}}$ per $1 \mathrm{~Gy}$. Moreover, because of the large sizes of $\mathrm{BD}$ and one type of track-etched detectors, seven specially designed PMMA slices were used to replace the existing ones in the $5 \mathrm{yr}$ old phantom.

\subsection{RS and compensator configuration}

To obtain proton energies suitable for treating shallow lesions, two types of pre-absorbers were used. The role of the pre-absorber is to degrade proton energies and shorten their path inside the patient's body. The RS dedicated for this purpose, included in the IBA Proteus- 235 therapy system with $70 \mathrm{MeV}$ minimal energy, is a uniform slab of Lexan $\left(\mathrm{C}_{16} \mathrm{H}_{14} \mathrm{O}_{3}, \rho=1.2 \mathrm{~g} \mathrm{~cm}^{-3}\right)$ material, with a thickness of $36.9 \mathrm{~mm}$ which corresponds to $41.96 \mathrm{~mm}$ water equivalent thickness and consequently 1.14 water equivalent ratio (WER). In the CCB facility, the RS is permanently attached to the nozzle, at a distance of $46 \mathrm{~cm}$ from the isocentre, and can only be positioned in or out of the beamline (figure 1).

The second pre-absorber used in the experiment was an individually designed, personalized 3D printed proton $\mathrm{BC}$. The $\mathrm{BC}$ is placed directly on the mask to which it adheres due to the exact modelling of its shape based on the patient's CT images. Thus, an air gap between the pre-absorber and the patient is avoided (figure 1).

For this experiment, individual compensators for two phantoms were printed. To be comparable to RS, the thickness of the compensators was set at $4 \mathrm{~cm}$ and polylactic acid, PLA $\left(\mathrm{C}_{3} \mathrm{H}_{4} \mathrm{O}_{2}, \rho=1.2 \mathrm{~g} \mathrm{~cm}^{-3}\right.$, Fiberlogy S.A.) thermoplastic material was selected, whose WER equals 1.18 as calculated from measurements. The Fused Filament Fabrication method was used for printing (Kuznetsov et al 2018). The first step is heating the material to the temperature at which it liquefies (for PLA $240{ }^{\circ} \mathrm{C}$ ) and the second step is printing the model layer by layer. A layer thickness of $0.25 \mathrm{~mm}$, a print path width of $0.8 \mathrm{~mm}$ and a nozzle diameter of $0.8 \mathrm{~mm}$ were used. In addition, the printout was air-cooled throughout the entire printing process.

12 The beam sigma is the single parameter characterizing the Gaussian shape. 

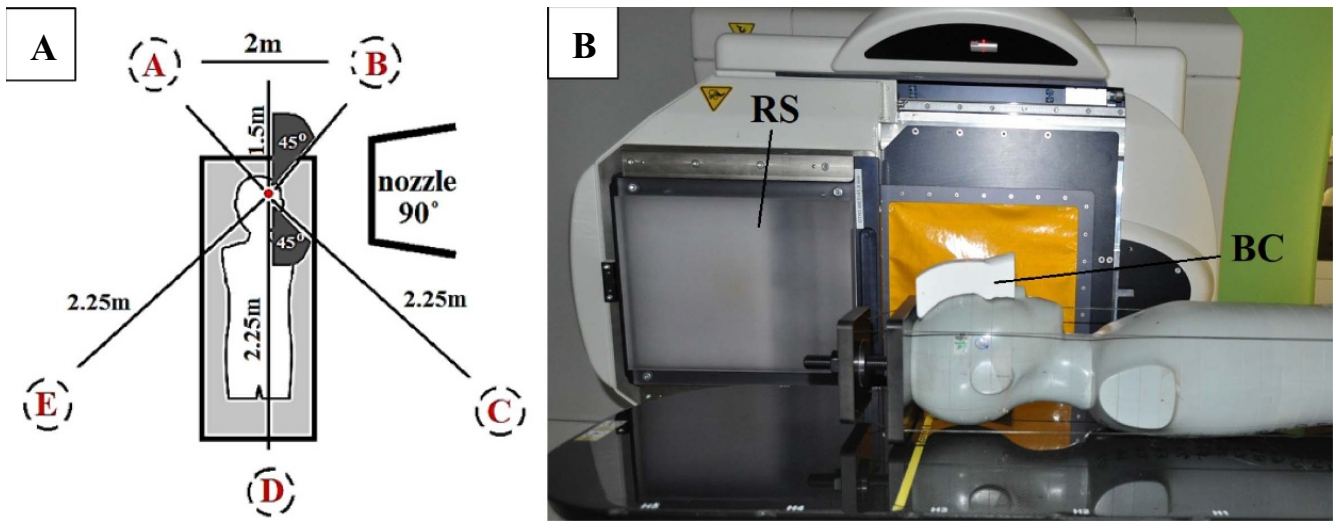

Figure 1. (A) Schematic view of active measurement positions (A)-(E) around the phantom with nozzle at $90^{\circ}$ position. (B) RS and $\mathrm{BC}$ configuration in the experiment.

\subsection{Experimental setup}

Phantoms were placed on the therapeutic table with its longer central axis perpendicular to the beam direction. Passive dosimeters were placed inside the phantoms in the dedicated plugs except for BD and one type of track-etched detector, which were placed in specially prepared PMMA slices. Active detectors measuring neutron ambient dose equivalent $H^{*}(10)$ were located at different angular positions around the phantom and at various distances from the isocentre described in table 1 in the supplementary data (available online at stacks.iop.org/PMB/66/035012/mmedia) and presented in figure 1 . There were no active detectors in the direction of the beam line. All detectors were located $1.25 \mathrm{~m}$ above the floor and aligned with the horizontal plane. The selected setup is similar to previous EURADOS WG9 experiments (Farah et al 2015, Mojżeszek et al 2017). Active measurements were performed in parallel with the exposure of passive detectors. Results were obtained for different doses, $D_{\mathrm{T}}$, i.e. 2, 40 and $100 \mathrm{~Gy}$ and renormalized to $1 \mathrm{~Gy}$.

\subsection{Active dosimetry inside the treatment room}

Neutron ambient dose equivalent $H^{*}(10)$ measurements were performed using five different neutron monitors presented in this section, which-except REM-2 and GW2 ionization chambers-are described in details in the previous EURADOS WG9 article (Stolarczyk et al 2018).

The REM-2 and GW2 chambers are cylindrical parallel-plate recombination chambers with an active volume of about $1800 \mathrm{~cm}^{3}$. Description and operation modes of REM-2 and GW2 were presented in detail by Zielczyński et al (2008) and Tulik et al (2018). The REM-2 is a chamber with a tissue equivalent electrodes filled with tissue equivalent gas mixture, classified as a recombination chamber. Because the REM-2 chamber approximates dosimetric parameters of the ICRU sphere (dose contribution and energy spectrum of secondary charged particles at depth of $10 \mathrm{~mm}), H^{*}(10)$ can be determined for mixed radiation fields (neutrons and photons) (Zielczyński et al 2008, Caresana et al 2014, Murawski et al 2018). To subtract the photon dose component, the twin-detector method was used (Golnik et al 2014) and as a companion to REM-2 the GW2 ionization chamber with aluminium electrodes and filled with $\mathrm{CO}_{2}$ was chosen. Due to its construction, GW2 is almost insensitive to neutrons. The experimental set-up was calibrated against an ${ }^{241} \mathrm{Am}$-Be source at the accredited calibration laboratory in National Centre for Nuclear Research (NCBJ). However, chambers were also tested in a high-energy reference radiation field (Mayer et al 2004), and energy response of both detectors were determined in monoenergetic neutron reference fields (Golnik et al 1997) in the National Physical Laboratory in Poland.

The Berthold LB 6411 (Burgkhardt et al 1997, Farah et al 2015) is a conventional $9.2 \mathrm{~kg}$ neutron meter with a central ${ }^{3} \mathrm{He}$ recoil proportional counter tube $(10 \mathrm{~cm}$ length, $4 \mathrm{~cm}$ external diameter) and moderating sphere of polyethylene ( $25 \mathrm{~cm}$ external diameter) and $\mathrm{Cd}$ absorbers.

The Wide Energy Neutron Detection Instrument second generation (WENDI-II) (Olsher et al 2000, Farah et al 2015) is an improved $13.5 \mathrm{~kg}$ survey meter with a central ${ }^{3} \mathrm{He}$ proportional tube counter surrounded by cylindrical boron-doped polyethylene moderator $(22.9 \mathrm{~cm}$ in diameter and $21 \mathrm{~cm}$ long). Extending the energy range up to $5 \mathrm{GeV}$ for neutrons was possible by using a tungsten powder shell $(1.5 \mathrm{~cm}$ in thickness) enclosing to the moderator.

Two Andersson-Braun neutron rem meters were used: a conventional NM2-458 and an extended-range NM2B-495Pb model (NE Technology Ltd). They consist of a cylindrical $\mathrm{BF}_{3}$ boron trifluoride proportional counter of $3.1 \mathrm{~cm}$ diameter and $7.2 \mathrm{~cm}$ active length surrounded by an inner moderating polyethylene layer 
( $1.7 \mathrm{~cm}$ thick), a $0.6 \mathrm{~cm}$ thick boron-doped synthetic rubber absorber and an outer polyethylene moderator $(6.9 \mathrm{~cm}$ thick). The extension of the energy range in the NM2B-495Pb model was possible by adding a $1 \mathrm{~cm}$ thick lead shell above the boron rubber.

The calibration factors for all instruments have been derived from irradiations with ${ }^{241} \mathrm{Am}-\mathrm{Be}$ source: the Berthold by the manufacturer, WENDI-II, REM-2 and GW2 at the accredited calibration laboratory in NCBJ and NM2B-458/NM2B-495Pb in Helmholtz Zentrum München.

The overall measurement uncertainty for all devices contains mainly the uncertainties in detector calibration, dose delivery, detector positioning and the energy response of the detectors. The last uncertainty should take into account the wide range of neutron energies encountered in PT (from thermal to fast neutrons), which together with the absence of neutron response functions for high energy neutron beams (up to $300 \mathrm{MeV}$ ) makes this component very difficult to quantify. Nonetheless, the overall relative measurement uncertainty is approximately $20 \%$ for the extended-range rem-counter (NM2B-495Pb) (Mares et al 2002, Farah et al 2015) and WENDI-II detector (Olsher et al 2000, Farah et al 2015) and 30\% for standard rem-meters (REM-2, Berthold and NM2B-458) (Burgkhardt et al 1997, Farah et al 2015).

Detectors at the various positions, with their uncertainties and energy responses are presented in table 1 in the supplementary data.

\subsection{Passive dosimetry methods used for in-phantom measurements}

Scattered photon beam radiation measurements were performed using radiophotoluminescence (RPL) detectors. Secondary neutrons were determined using polyallyldiglycol carbonate based track-etched (PADC) detector and BD, while the thermal neutron component was measured with a set of two types of thermoluminescent (TL) detectors. Basic principles, characteristic and calibration procedures of these passive dosimetry methods were described in previous EURADOS WG9 papers (Knežević et al 2013, Miljanić et al 2014, Stolarczyk et al 2018), but relevant information can be also found below. Types of detectors, institutions that provided them and combined relative uncertainties are presented in table 2 in the supplementary data.

\subsubsection{Luminescence detectors used for out-of-field photon and neutron dosimetry}

Two types of TL detectors in the form of pellets were used: MTS-7 ( $\left.{ }^{7} \mathrm{LiF}: \mathrm{Mg}, \mathrm{Ti}\right)$ and MTS-6 ( $\left.{ }^{6} \mathrm{LiF}: \mathrm{Mg}, \mathrm{Ti}\right)$, both of which are based on a lithium fluoride matrix with magnesium dopants. All TL detectors were calibrated using a ${ }^{60}$ Co source. The net difference between the readings of MTS- 6 and MTS-7 was used to calculate the gamma-equivalent neutron doses, $D_{\mathrm{n}}$. RPL detectors (type GD-352M, manufacturer AGC Techno Glass) in the form of small glass rods were used for photons measurements. Detectors used during this experiment also contain an energy compensation filter and can be used for out-of-field dose measurements, where scattered photons predominate. RPLs were calibrated in terms of kerma free-in-air, $K_{\text {air }}$, using a ${ }^{60}$ Co source and then converted to $D_{\mathrm{w}}$ using experimentally determined factors (Knežević et al 2013). For determining the uncertainty of dose calculation for TLDs and RPLs, the same procedures were used as presented in Knežević et al $(2013,2018)$ and Stolarczyk et al (2018). Particular calculated uncertainties are presented in the table 2 in the supplementary data.

\subsubsection{Track-etched and BD used for out-of-field neutron dosimetry}

Nuclear Physics Institute Czech Academy of Sciences (NPI CAS) used PADC based $\left(\mathrm{C}_{12} \mathrm{H}_{18} \mathrm{O}_{7}\right)$ track-etched detectors type HARZLAS TD-1 (Nagase Landauer Ltd, Japan) with density $1.3 \mathrm{~g} \mathrm{~cm}^{-3}$. Detectors were cut into $17 \mathrm{~mm}$ wide strips thick $0.9 \mathrm{~mm}$. The neutron dose equivalent $H$ was calculated based on the relationship between the parameters of etched tracks and the particles' LET (Jadrnícková et al 2008, Pachnerová Brabcová et al 2013). Associated uncertainties include statistical uncertainties related to the counting of particle tracks in different LET bins and systematic errors associated with uncertainties in calibration curves. Here we combine both sources of uncertainty which lead to a total uncertainty of $\sim 20 \%$. The uncertainty does not include the angular dependence of the track detector's response (Ambrožová et al 2019).

Universitat Autònoma de Barcelona (UAB) has developed a dosimeter based on PADC track-etched detectors (García et al 2005, Domingo et al 2013) with a layered structure described in detail by Domingo et al (2013) and García et al (2005). These detectors were previously used in EURADOS WG9 experiments and the procedure for reading the dose, based on MC simulation, was presented by Stolarczyk et al (2018) and García-Fusté et al (2020). The neutron dose equivalent $H$ was computed according to the Kerma Approximation (therefore using the neutron fluence-to-kerma conversion coefficients $k_{\Phi}(E)$ in ICRU 4-component tissue substitute (Chadwick et al 1999) and using the neutron quality factors $Q(E)$ (Siebert and Schuhmacher 1995)). From the experimental net track density per Gy, $C_{\text {PADC }}$, the response function of the whole dosimeter in terms of fluence $R_{\Phi}(E)$ (Domingo et al 2013) and the MC unit spectral neutron fluence, 
$\varphi_{\mathrm{E}}(E)$. Based on that quantities, neutron dose equivalent, $H$, and neutron fluence, $\Phi_{\mathrm{PADC}}$, can be evaluated. This methodology has been already used and described by García-Fusté et al (2020). The main source of uncertainty comes from the experimental uncertainty associated with the statistical counting of radiation in the PADC layer. The uncertainties of the unit spectral neutron fluence come purely from the MC statistical procedure. Relative uncertainties on the evaluated neutron dose equivalent ranges from $7 \%$ (near the proton field) to $60 \%$ (far from the proton field).

$\mathrm{BD}$, used for personal neutron dosimetry (Bubble Technology Industries) were used to measure neutrons with energies above $200 \mathrm{keV}$. They are characterized by zero sensitivity to photons and are independent of dose rate and energy response. Calibration was performed in a ${ }^{252} \mathrm{Cf}$ field in the Secondary Standard Dosimetry Laboratory of SCK CEN and applying the fluence-to-dose equivalent conversion factors obtained from kerma factors for ICRU tissue (Siebert and Schuhmacher 1995). The uncertainty in the calibration and therefore also the number of bubbles per dose equivalent is $20 \%$, but also the positioning uncertainty of both the phantom and detector should be taken into account, which is estimated at a maximum of $5 \%$. By combining the above sources, a total uncertainty of $25 \%$ was determined.

\subsection{MC simulations}

The aim of the MC simulations was to calculate the unit neutron spectral fluence $\varphi_{\mathrm{E}}(E)$, at the positions of the PADC (UAB) detectors in the phantoms. which are required to calculate neutron dose equivalent from the readings of the detector. MC simulations were performed using MCNPX (version 2.7.0) (Pelowitz 2011). Composition and densities of the tissues of the voxelized phantom were taken from ICRU Report-44 report (White et al 1989). A simplified beam model in the form of a parallel proton beam emitted from a disk of $6 \mathrm{~cm}$ in diameter was applied for calculation. In total 25 and 26 proton energies ranging from 80 to $140 \mathrm{MeV}$ were used for BC and RS, respectively. The source was located $46 \mathrm{~cm}$ from the isocentre inside the phantom surrounded by air. In addition to pre-absorbers, no other room details were considered-their contribution to the total neutron dose is not significant, which was confirmed by Mares et al (2016) through the spectrometric in-room measurements where thermal neutrons contributed to $7 \%-24 \%$ of total fluence but only $2 \%$ to dose equivalent. Unit neutron spectral fluence $\varphi_{\mathrm{E}}(E)$ were determined in spherical volumes of $0.5 \mathrm{~cm}$ diameter at the corresponding detector positions inside the phantom. In the simulations, no detectors were placed at positions, so the disturbance of the neutron field were not included.

The cross sections libraries used in the MCNPX radiation transport were selected automatically according to the pre-defined options in XSDIR file evaluated in 2013. Proton cross sections up to a proton incident energy of $150 \mathrm{MeV}$ were taken from ENDF/B-VII (.70h) (Chadwick et al 2006), but applied only for pre-absorbers and their main components (C-12, O-16 and $\mathrm{H}-1)$. Because the voxelized phantoms in simulations were represented by its natural composition, not the most abundant isotopes, inside the phantom for the proton transport and neutron production the default configuration of the MC code was employed (Bertini (Valentin 2003, Stolarczyk et al 2011) + pre-equilibrium model + Dresner (1981) with RAL (Atchison, 1980) fission evaporation model). For all substances containing hydrogen inside the phantom, the neutron thermal scattering effects due to $\mathrm{H}-1$ are taken into account through the sublibrary endf70sab (lwtr.10t), which also belongs to the library ENDF/B-VII.

Only transport of neutrons, protons and photons has been considered to be relevant when scoring the unit neutron spectral fluence inside the phantom. Photonuclear reactions, as well as light ion rejection from neutrons and protons were employed in simulations.

\section{Results}

All results are presented in $\mu \mathrm{Gy}$ or $\mu \mathrm{Sv}$ and normalized to the proton dose deposited in the centre of the target, i.e. at the isocentre. Error bars were not included in plots of experimental results, for clarity.

\subsection{Neutron ambient dose equivalent $H^{*}(10)$ inside the treatment room}

Mapping of stray neutron ambient dose equivalent $H^{*}(10)$ was performed inside the treatment room. Table 1 presents results as mean values of neutron dose equivalent normalized to total dose, $H^{*}(10) / D_{\mathrm{T}}$ $\left(\mu \mathrm{Sv} \mathrm{Gy}^{-1}\right)$ measured for each field and phantom type on every position inside the treatment room. The highest and the lowest values of $1.77 \mu \mathrm{Sv} \mathrm{Gy}^{-1}$ in position $\mathrm{B}$ and $0.37 \mu \mathrm{Sv} \mathrm{Gy}^{-1}$ in position D were noticed for the $5 \mathrm{yr}$ old phantom, nozzle angle $0^{\circ}$ with RS as pre-absorber, and $10 \mathrm{yr}$ old phantom, nozzle angle $90^{\circ}$ with $\mathrm{BC}$ as pre-absorber, respectively. Generally, for all measurements, the lowest dose was measured in position $\mathrm{D}$, while the highest doses were observed at both positions $\mathrm{A}$ and $\mathrm{B}$, for nozzle angles $90^{\circ}$ and $0^{\circ}$, respectively. In most of the positions doses determined for RS irradiations are systematically higher than for BC by $20 \%-30 \%$ but the uncertainty of measurements are of the same order. Moreover, higher ratios were 


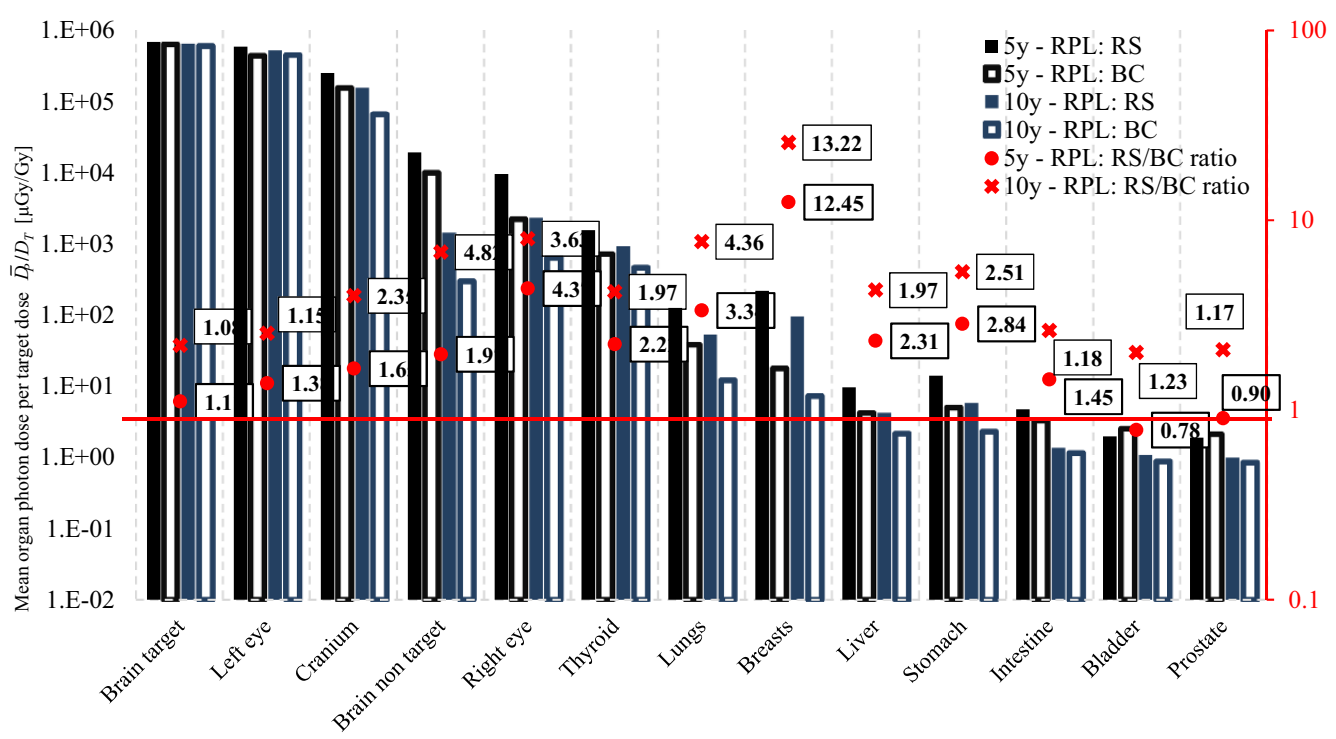

Figure 2. Mean organ photon doses per target dose $\bar{D}_{\mathrm{P}} / D_{\mathrm{T}}$ and the ratios of RS/BC doses for RPL detectors for 5 and 10 yr old phantom after irradiation with BC and RS. The exact values of the organ doses are presented in tables 3 and 4 in the supplementary data. The uncertainties can be calculated as the uncertainty of the average value using values for particular detectors from table 2 in the supplementary data.

observed for irradiations at the $90^{\circ}$ nozzle position especially at positions B ( 1.69 vs. 1.08 for 5 and $10 \mathrm{yr}$ phantoms) and D (1.46 vs. 1.12 and 1.52 vs. 1.20 for 5 and $10 \mathrm{yr}$ phantom, respectively).

\subsection{Out-of-field photon doses inside the phantoms}

The photon doses $D_{\mathrm{P}}$ were measured by RPL detectors. Mean organ doses per target dose $\bar{D}_{\mathrm{P}} / D_{\mathrm{T}}(100 \mathrm{~Gy})$ and the ratios of RS/BC doses for both phantoms are presented in figure 2 . The exact values of the organ doses are presented in tables 3 and 4 in the supplementary data. The mean organ doses were calculated as the arithmetic mean of doses from all detectors placed in the volume of the given organ. In most cases higher organ doses for RS compared to BC are observed, especially for breasts and lungs. The opposite situation is observed only for some distant organs as bladder and prostate in the $5 \mathrm{yr}$ old phantom. For almost all organs, $\mathrm{RS} / \mathrm{BC}$ ratios are higher than 1, obtaining the highest ratio for breasts in both phantoms.

Out-of-field photon doses per target dose $D_{\mathrm{p}} / D_{\mathrm{T}}$ as a function of distance from the isocentre for both phantoms are presented in figure 3. The graphs show that regardless of the distance from the isocentre, the doses of out-of-field photons are lower for BC than for RS. For both types of detectors and pre-absorbers, a similar trend can be observed, showing a reduction in photon doses by almost six orders of magnitude from positions close to $5 \mathrm{~cm}\left(\sim 6 \mathrm{E}+05 \mu \mathrm{Gy} \mathrm{Gy}^{-1}\right)$ and up to $50 \mathrm{~cm}$ from the isocentre $\left(\sim 2-3 \mu \mathrm{Gy} \mathrm{Gy}^{-1}\right)$. In the 5 and $10 \mathrm{yr}$ phantoms the maximum of RS/BC ratios occurs at different distances from the isocentre due to the different sizes of the phantoms.

\subsection{Out-of-field neutron dose equivalent inside the phantoms}

The neutron dose equivalent $H$ was measured by BD, by a set of MTS-6/MTS-7 detectors and two types of track-etched detectors. Figure 4 shows the neutron dose equivalent per target dose $H / D_{\mathrm{T}}$ and the ratios of $\mathrm{RS} / \mathrm{BC}$ doses at different points and distances from the isocentre for the $5 \mathrm{yr}$ old phantom, measured by BD and PADC from UAB. The exact values of the organ doses are presented in table 5 in the supplementary data.

For these measurements it was necessary to use appropriate PMMA slices to hold the detectors. The position number on the chart corresponds to the number of the replaced slice in the phantom, while letters F, $\mathrm{B}, \mathrm{R}, \mathrm{L}$ and $\mathrm{M}$ indicate the position in the slice and mean: front, back, right, left and middle, respectively. Except for two positions-15FR for PADC and 17FM for BD-in all other positions, higher doses for RS in comparison with $\mathrm{BC}$ were observed. The highest $\mathrm{RS} / \mathrm{BC}$ ratios at the distance between 20 and $30 \mathrm{~cm}$ from the isocentre for both types of detectors are observed (10.2 and 2.6 for PADC and BD, respectively). At the furthest three positions for irradiation with $\mathrm{BC}$ as the pre-absorber, the PADC detectors were under the detection limit.

Results of TLD measurements for $5 \mathrm{yr}$ old phantom are presented in figure 2 in the supplementary data as the gamma-equivalent neutron dose per target dose $D_{\mathrm{n}} / D_{\mathrm{T}}$. It was calculated as the net difference between the readings of MTS- 6 and MTS-7 detectors calibrated in terms of response to Co- 60 gamma-rays. This 


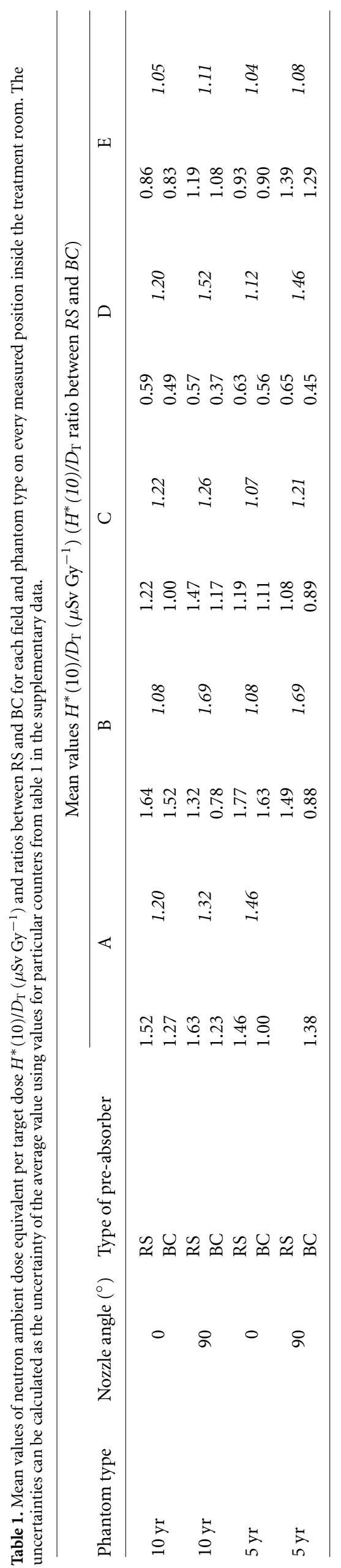



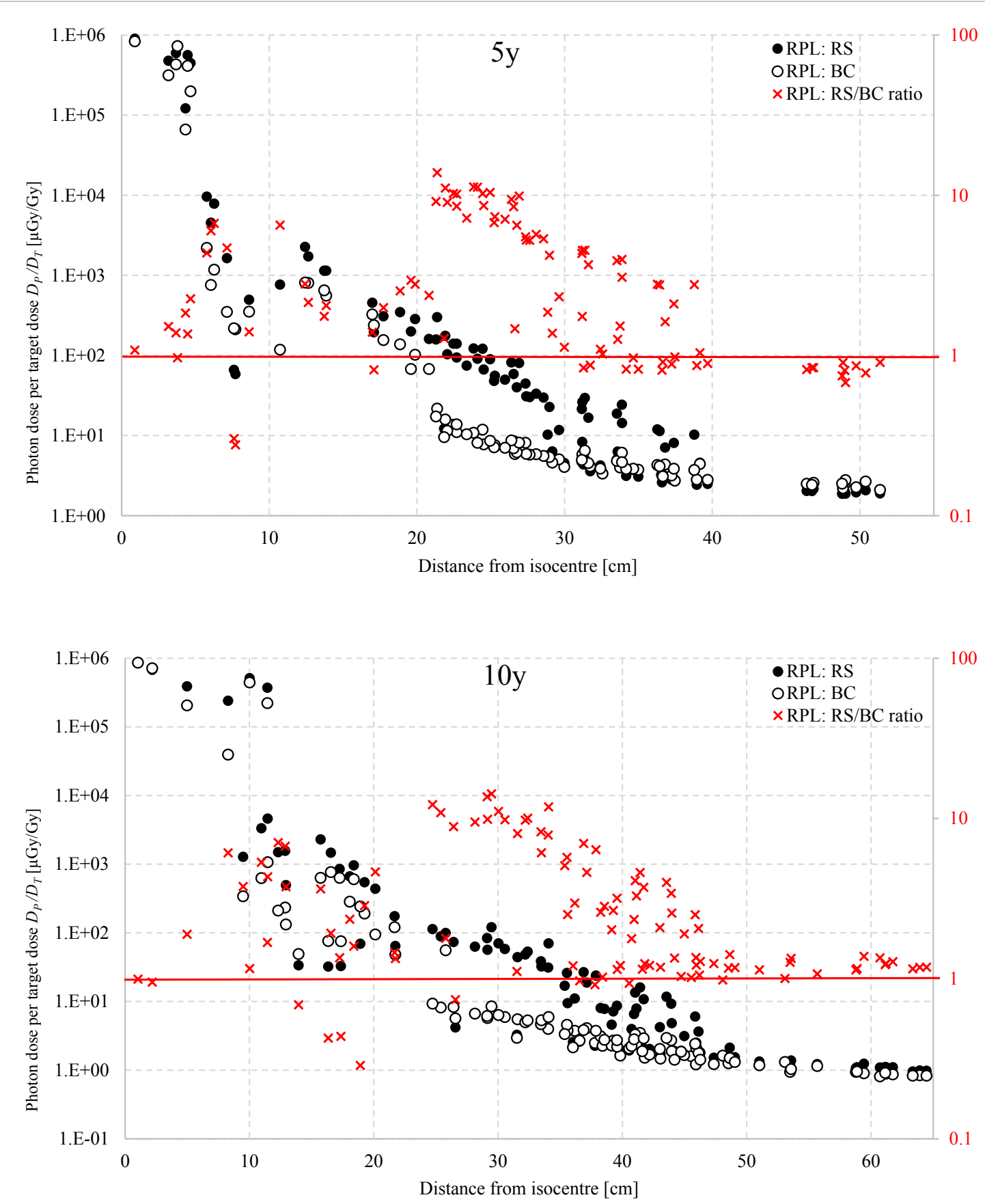

Figure 3. Photon dose per target dose $D_{\mathrm{P}} / D_{\mathrm{T}}$ and the ratios of RS/BC doses measured with RPL detectors in 5 (top) and $10 \mathrm{yr}$ (bottom) old phantom as a function of distance from the isocentre. The uncertainties can be assessed using values for particular detectors from table 2 in the supplementary data.

difference is proportional to the thermal neutron fluence at the position of the detector (Stolarczyk et al 2018). The dose values obtained in this way require a conversion to be comparable with neutron doses obtained by reading BD (see section 4.1. The correlation between gamma-equivalent neutron dose and dose equivalent at the Discussion part).

Figure 5 shows mean neutron dose equivalent in organs per target dose $\bar{H} / D_{\mathrm{T}}$ measured with PADC detectors for $10 \mathrm{yr}$ old phantom, whilst the same results as a function of distance from the isocentre $H / D_{\mathrm{T}}$ are presented in figure 6. The exact values of the organ doses are presented in table 6 in the supplementary data. It has been observed that for organs located near the isocentre, the measured neutron doses are higher for RS than BC (e.g. for breasts 3.7 times), but for organs located at a greater distance, the relationship is reversed. This is particularly visible on the graph showing doses as a function of distance from the isocentre-from a distance of about $35 \mathrm{~cm}$ to over $60 \mathrm{~cm}$, doses for BC are higher (for gallbladder 4.4 times or for intestine 3.8) compared to RS. For organs closer to the isocentre, the measured doses were lower for $\mathrm{BC}$. The highest $\mathrm{RS} / \mathrm{BC}$ ratio for the distance between 20 and $40 \mathrm{~cm}$ from the isocentre are observed with a maximum ratio of 


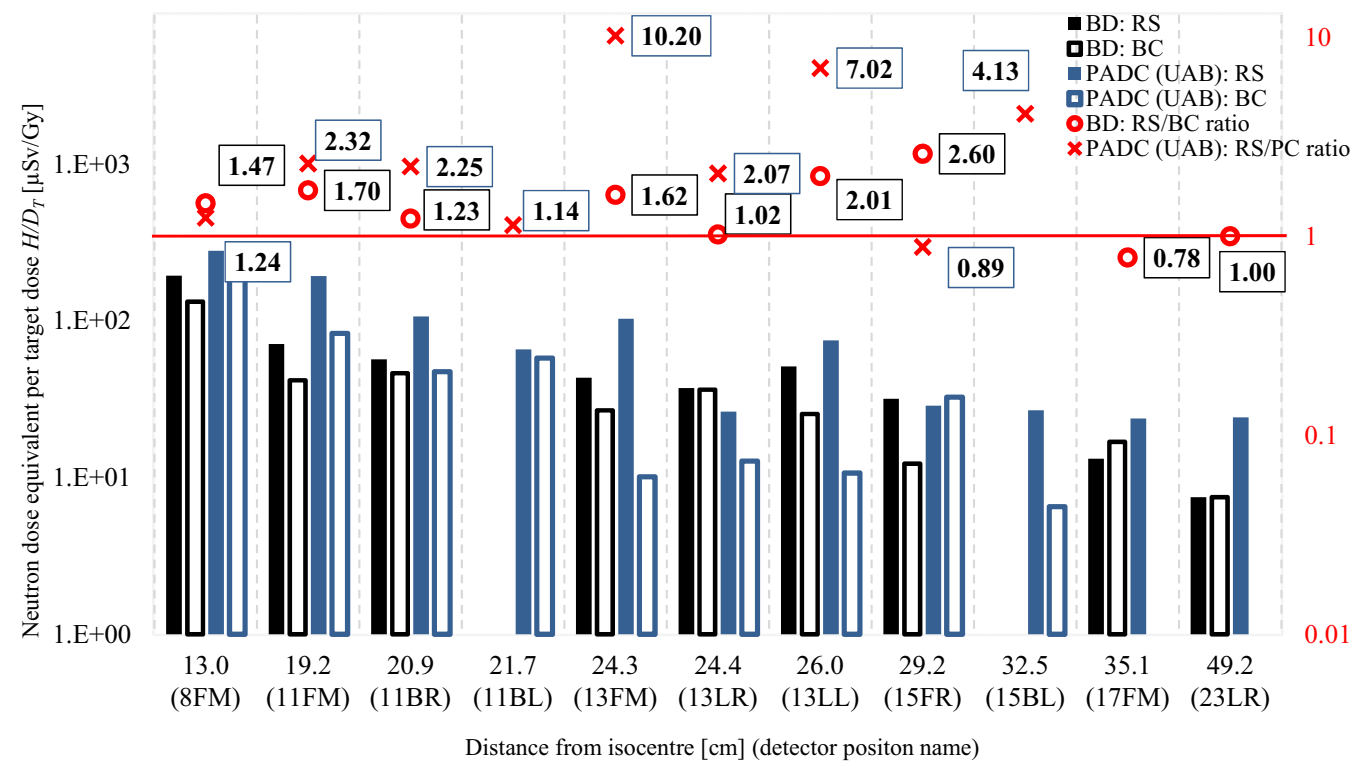

Figure 4. Neutron dose equivalent per target dose $H / D_{\mathrm{T}}$ and the ratios of $\mathrm{RS} / \mathrm{BC}$ doses in particular position and distances from the isocentre measured with BD and PADC (UAB) for RS and BC in the $5 \mathrm{yr}$ old phantom. The exact values of the organ doses are presented in table 4 in the supplementary data. The uncertainties can be assessed using values for particular detectors from table 2 in the supplementary data.

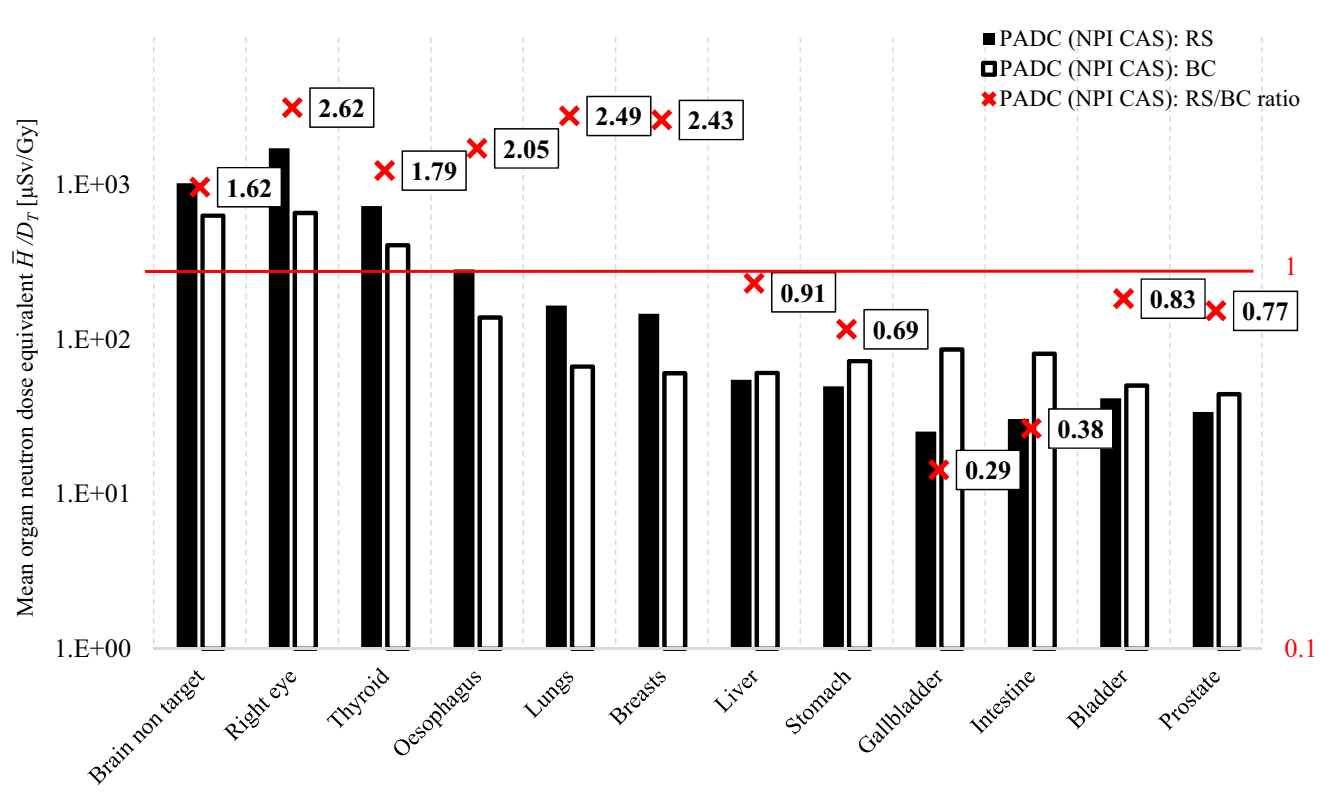

Figure 5. Mean neutron dose equivalent in organs per target dose $\bar{H} / D_{\mathrm{T}}$ and the ratios of RS/BC doses for PADC (NPI CAS) for $\mathrm{RS}$ and $\mathrm{BC}$ in the $10 \mathrm{yr}$ old phantom. The exact values of the organ doses are presented in table 6 in the supplementary data. The uncertainties can be calculated as the uncertainty of the average value using values for particular detectors from table 2 in the supplementary data.

10.6. Such distances correspond to the positions of the lungs, breasts, liver and-for the 5 yr old phantom only—stomach and intestine. The ratios at distances further than about $40 \mathrm{~cm}$ from the isocentre (which corresponds to the positions of the liver, stomach, gallbladder, intestine, bladder and prostate) decreases below unity. However, for such large distances the measured doses are relatively low (from 34.5 to $\left.119.2 \mu \mathrm{Gy} \mathrm{Gy}^{-1}\right)$.

\subsection{MC simulations}

The unit neutron spectral fluence $\varphi_{\mathrm{E}}(E)$ was calculated with MC simulations at the positions of the PADC (UAB) detectors in the $5 y$ old phantom. Figure 7 shows the differences in the spectral neutron fluence, $\Phi_{\mathrm{E}}(E)=\Phi_{\mathrm{PADC}} \cdot \varphi_{\mathrm{E}}(E)$, where $\Phi_{\mathrm{PADC}}$ is the evaluated neutron fluence (see section 2.6) normalized to 1 Gy of 


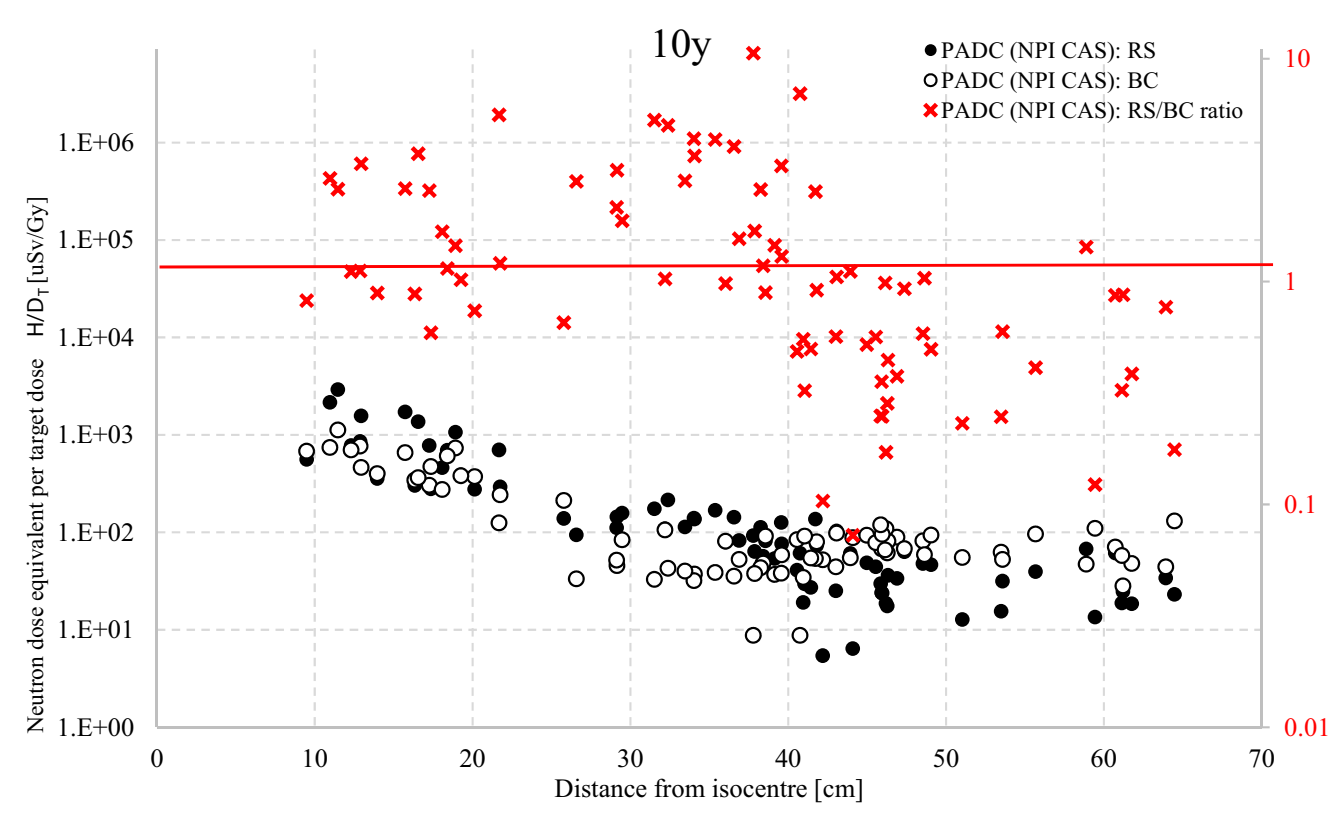

Figure 6. Neutron dose equivalent per target dose $H / D_{\mathrm{T}}$ for PADC (NPI CAS) for RS and BC and the ratios of RS/BC doses in the $10 \mathrm{yr}$ old phantom as a function of distance from the isocentre. The uncertainties can be assessed using values for particular detectors from table 2 in the supplementary data.

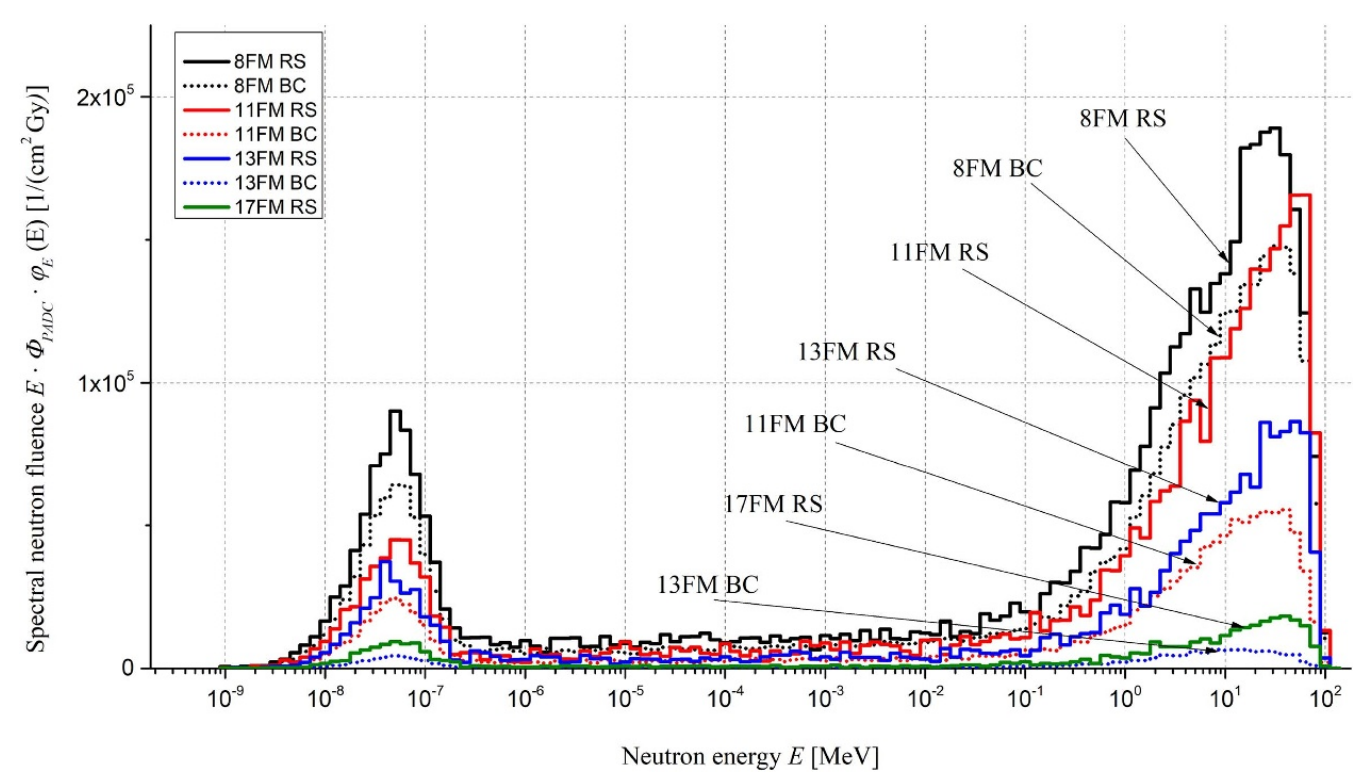

Figure 7. Spectral neutron fluence, $\Phi_{\mathrm{E}}(E)=\Phi_{\mathrm{PADC}} \cdot \varphi_{\mathrm{E}}(E)$ normalized to the unit dose at the isocentre in four positions inside the $5 y$ phantom (8FM, 11FM, 13FM and 17FM, see section 3.3) calculated with MC simulations.

the dose in the isocentre (40 Gy in total, based on measurements). Results present the spectral neutron fluence for RS and BC at four different detector positions, at distances: 13.0 (8FM), 19.2 (11FM), 24.3 $(13 \mathrm{FM})$ and $35.1 \mathrm{~cm}(17 \mathrm{FM})$ (abbreviations of positions names are described in section 3.3). It can be observed that the contribution of thermal neutrons in the RS spectrum is bigger than in the BC spectrum at each distance. Thermal neutrons do not significantly increase the dose equivalent in tissue, but they predominately contribute to the response of MTS- 6 detectors containing the Li- 6 isotope. Moreover, the RS spectra show more high-energy neutrons generated at greater distances, than BC, which leads to higher doses.

To better illustrate the impact of geometry on the secondary neutrons measured inside the phantom, from a subset of the total MC simulations we obtain a visualization of paths of neutrons, produced in pre-absorber materials, was generated for the $0^{\circ}$ nozzle angle (figure 8 ). The colour version allows secondary neutrons to be distinguished according to their energy and shows that more high energy neutrons reach the phantom and the critical organs volumes while using RS, than BC. 


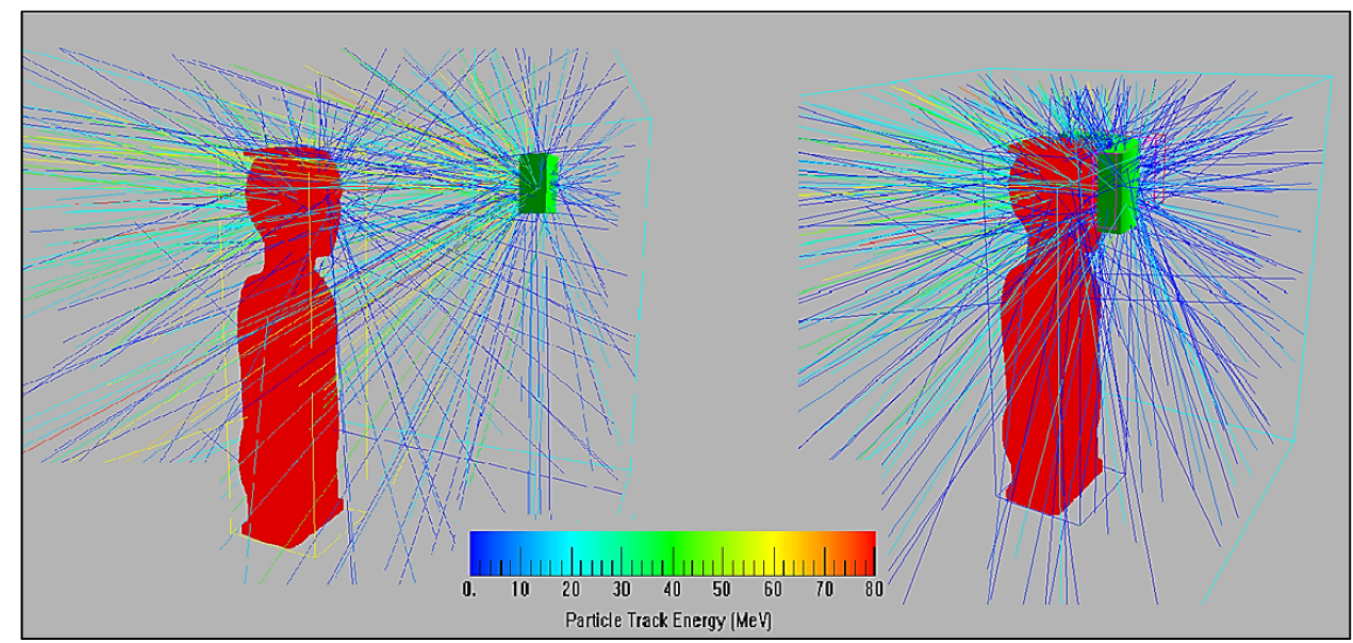

Figure 8. Visualization of paths of neutrons, produced in two pre-absorbers (left-RS, right-BC), for the $0^{\circ}$ nozzle angle.

\section{Discussion}

In this section the obtained results and the correlations between them are discussed. Additionally, the reproducibility of measurements of active detectors were verified and the results are presented in the supplementary data.

\subsection{The correlation between neutron ambient dose equivalent $H^{*}(10)$ and position around the phantom} Results of active measurements are presented for both pre-absorbers in table 1 . In most of the positions doses determined for RS irradiations are systematically higher than for BC by $20 \%-30 \%$. This is because neutrons produced in the compensator are directly attenuated in the phantom and deliver lower doses to distant organs. In contrast, the RS placed $46 \mathrm{~cm}$ from the isocentre produces a neutron shower which leads to exposure of the entire phantom. Higher ratios of RS/BC values were observed for irradiations at the $90^{\circ}$ nozzle position especially at positions B (1.69 vs. 1.08 for 5 and $10 \mathrm{yr}$ phantoms) and D (1.46 vs. 1.12 and 1.52 vs. 1.20 for 5 and $10 \mathrm{yr}$ phantom, respectively). At position $\mathrm{B}$, where the differences are the largest, it should be noticed, that for the nozzle angle $0^{\circ}$ the detector recorded twice higher dose for BC, while for RS only higher by about $20 \%$ comparing with the doses detected for nozzle angle $90^{\circ}$. This disproportionality may be caused by the fact that while the nozzle is at the angle $90^{\circ}$ the gantry arm partly covered the detector and crossed the neutron path line between the compensator and the detector, thus absorbing and scattering some neutrons. Higher doses were also observed at positions $\mathrm{C}$ and $\mathrm{E}$ than at position $\mathrm{D}$, although these detectors are located at the same distance from the isocentre. At positions $\mathrm{C}$ and $\mathrm{E}$, detectors with extended energy range were positioned, while the detector on position $\mathrm{D}$ detects neutrons in the energy range from thermal to $14 \mathrm{MeV}$ (see table 1 in the supplementary data). Detectors with an extended energy range absorb neutrons with energy higher than $14 \mathrm{MeV}$, which resulted in a higher neutron dose equivalent. It is also worth emphasizing that the dose equivalent at position $\mathrm{D}$ is around $25 \mu \mathrm{Sv}$ per 50 Gy target dose during the entire treatment course.

\subsection{Comparison of out-of-field doses measured with $\mathrm{RS}$ and $\mathrm{BC}$}

A comparative analysis of the results for RS and $\mathrm{BC}$ was made by presenting the ratios RS/BC for both photon and neutrons doses. Significantly higher photon organ doses were observed for the breast using RS (figure 2) than BC, which may result from the superficial location of the detectors-the dose for the breast is recorded by two shallowly located dosimeters placed at chest height. A secondary photons shower generated in the RS reaches the phantom surface through the air gap, while that generated in the BC immediately interacts with subsequent internal phantom structures, thus limiting the possibility of wide-angle dispersion. A similar relationship is observed at many points located close to phantom surfaces at a distance from 20 to $40 \mathrm{~cm}$. Considering the previously mentioned effect of the air gap, it can be concluded that a higher ratio of $\mathrm{RS} / \mathrm{BC}$ doses is observed at a closer position of the detector relative to the phantom surface. It is worth noting that the calculated distance from the isocentre does not provide unambiguous information about the location of the measuring point relative to the phantom edges - a distance of $30 \mathrm{~cm}$ from the isocentre may correspond to a detector located both at a depth of 10 and $2 \mathrm{~cm}$. Similar results, i.e. the highest ratios of 


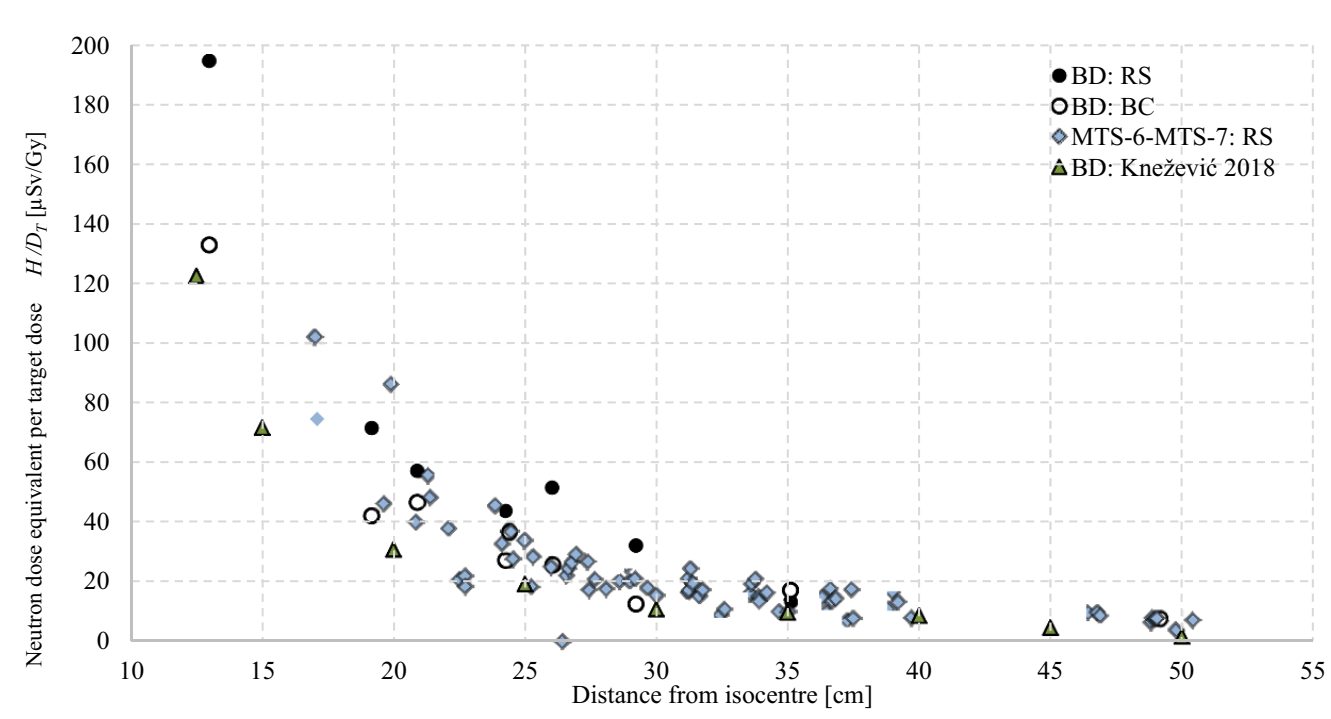

Figure 9. Neutron dose equivalent per target dose $H / D_{\mathrm{T}}$ measured with bubble detectors $\mathrm{BD}$ and neutron dose equivalent derived from the TLDs measurement (MTS-6-MTS-7) for $5 \mathrm{yr}$ old phantom as a function of distance from the isocentre.

$\mathrm{RS} / \mathrm{BC}$ doses for the distance between 20 and $30 \mathrm{~cm}$ from the isocentre are observed for secondary neutrons (figures 4 and 6). Such distances correspond to the positions of the lungs, breasts, liver and-for the 5 yr old phantom only_stomach and intestine. This may be again due to the lower attenuation of secondary neutrons in the air gap before reaching the phantom, as compared to BC. For neutron doses recorded by PADC detectors from NPI CAS, it has been observed that ratios of RS/BC doses at distances further than about $40 \mathrm{~cm}$ from the isocentre (which corresponds to the positions of the liver, stomach, gallbladder, intestine, bladder and prostate) decrease below unity. However, for such large distances the measured doses are relatively low (from 34.5 to $119.2 \mu \mathrm{Sv} \mathrm{Gy}^{-1}$ ), so their influence on exposure of children is much lower.

\subsection{The correlation between gamma-equivalent neutron dose and dose equivalent}

Pairs of ${ }^{6} \mathrm{LiF} /{ }^{7} \mathrm{LiF}$ thermoluminescence detectors are used in dosimetry to discriminate between neutron and gamma-ray doses. The difference in response between ${ }^{6} \mathrm{LiF}$ and ${ }^{7} \mathrm{LiF}$ detectors, caused by the higher ${ }^{6} \mathrm{Li}$ cross section in the $\operatorname{Li}(n, \alpha)$ T reaction, is expressed in terms of the gamma-equivalent neutron dose, $D_{n}$ (Knežević et al 2018). However, this quantity cannot be used to infer neutron dose equivalent because the dose in tissue is predominately produced by high energy neutrons whereas ${ }^{6} \mathrm{Li}$ reacts mainly with thermal neutrons. In a previous EURADOS experiment, dosimetric measurements of a scattered proton beam were performed in a $30 \mathrm{~cm} \times 30 \mathrm{~cm} \times 60 \mathrm{~cm}$ water phantom using PADC, calibrated in terms of neutron dose equivalent and pairs of MTS-6/MTS-7 detectors (Stolarczyk et al 2018). The conversion coefficient relating the PADC (UAB) detectors and net signal of TLD varied between 0.3 and $0.7 \mathrm{mSv} \mathrm{mGy}^{-1}$, depending on the position in the phantom. For the current measurements, the best agreement with the readouts of PADC was obtained for the conversion coefficient $0.9 \mathrm{mSv} \mathrm{mGy}^{-1}$. The presence of pre-absorbers changes the neutron energy spectrum, which has an impact on the conversion coefficient. For such converted results, a comparison with $\mathrm{BD}$ and results from a previous experiment with the same conditions (but without a pre-absorber) from Knežević et al (2018) is presented in figure 9. As expected, neutron doses are lowest for irradiation without any pre-absorber.

\subsection{Understanding neutron energy spectra}

The spectral neutron fluence presented in figure 7 depends on the position of the pre-absorber relative to the phantom. Secondary neutrons are produced and spread in the pre-absorber material. Due to the fact, that the RS is $46 \mathrm{~cm}$ from the target, while the $\mathrm{BC}$ is on the phantom surface, the angles between the pre-absorber, as the source of the neutron beam, and the detectors located inside the phantom are about $10^{\circ}-40^{\circ}$ for RS and almost $80^{\circ}-90^{\circ}$ for $\mathrm{BC}$. Therefore, in such geometry more neutrons can be observed inside the phantom for RS than for BC. This impact of the geometrical set up is presented in figure 8. Moreover, the colour coding of this figure shows, that high energy neutrons (above $10 \mathrm{MeV}$ ) are produced in the forward direction, while low energies neutrons (below $10 \mathrm{MeV}$ ) appear to be isotropic. This leads to conclusions that more of high energy neutrons reach the phantom and the critical organs volumes while using RS, than BC, because of the larger distance from the phantom surface. 


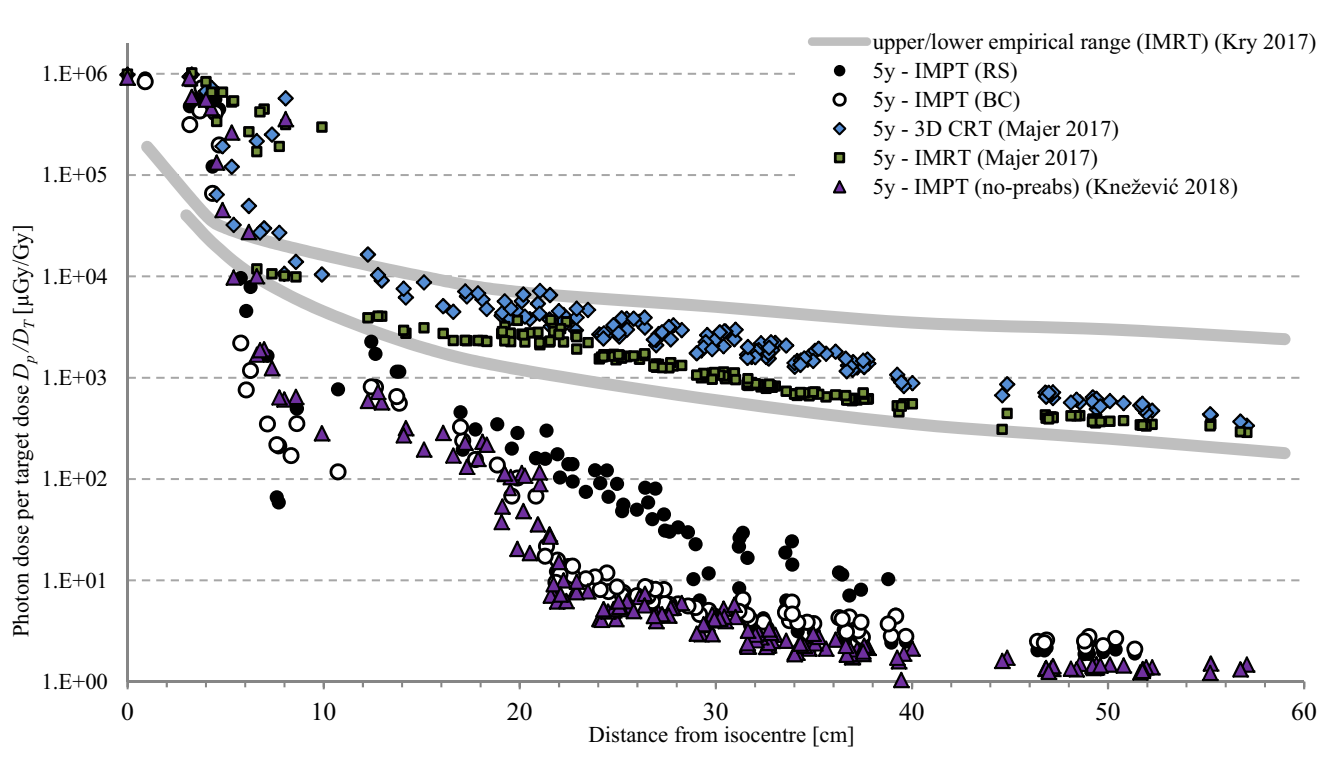

Figure 10. Out-of-field photon dose per target dose $D_{\mathrm{p}} / D_{\mathrm{T}}$ inside the phantom measured by RPL detectors for all three experiments (Majer et al 2017, Knežević et al 2018) for $5 \mathrm{yr}$ old phantom presented as a function of distance from the isocentre. The upper and lower bounds (solid curves) presented the empirical range of doses associated with different IMRT techniques, presented by Kry et al (2017).

\subsection{Comparison with data from literature}

The results were compared with the literature data presented in Majer et al (2017) and Knežević et al (2018) collected in other EURADOS WG9 experiments with the same phantoms, tumour size and locations. In Majer et al (2017) two different treatment techniques based on $\mathrm{x}$-ray radiotherapy, i.e. 3D conformal radiation therapy (3D CRT) and intensity modulated radiation therapy (IMRT) were used, while in Knežević et al (2018) the experiment was performed in the same PT facility (CCB IFJ PAN), so intensity modulated PT (IMPT) treatment technique was used. The results showed by Knežević et al (2018) represent cases of these paediatric tumours, such as base-of skull tumours, which are situated deeper and should be treated without pre-absorbers to avoid an additional beam scattering. The target diameter in all experiment was the same $-6 \mathrm{~cm}$. Out-of-field photon doses per target dose $D_{\mathrm{P}} / D_{\mathrm{T}}$ inside the phantom measured by RPL detectors for all three experiments for a $5 \mathrm{yr}$ old phantom are presented as a function of distance from the isocentre in figure 10 . In the vicinity of the isocentre, i.e. at a distance of 10-15 cm, doses from secondary photons were recorded at a similar level (excluding doses for 3D CRT), while at a depth of $30 \mathrm{~cm}$, secondary doses for IMRT and 3D CRT are one and two orders of magnitude higher than for RS and BC, respectively. Both doses for 3D CRT and IMRT are close to IMPT only in the vicinity of the isocentre, while at a distance of about $10 \mathrm{~cm}$ doses are higher by one and even two orders of magnitude at further distances, especially comparing to IMPT with the $\mathrm{BC}$ as a pre-absorber. Moreover, comparison with IMPT irradiation without any type of a pre-absorber (Knežević et al (2018)) showed higher photon doses at a distance from 20 to $40 \mathrm{~cm}$ for irradiations with RS, remaining comparable for closer and further distances. Doses for irradiation with the use of a BC can be considered, within the limits of uncertainty, to be consistent with doses obtained without a pre-absorber, but a slight but systematic shift and thus lower doses for irradiations without a pre-absorber can be observed. Similar relationships were observed for a $10 \mathrm{yr}$ old phantom (not presented in the figure 10, for clarity). The obtained results were also compared with the empirical range of doses associated with different IMRT techniques, presented by Kry et al (2017).

Neutron dose equivalent was also measured by Knežević et al (2018) using the same type of detectors-BD and PADC for a $5 \mathrm{yr}$ old phantom. Such results were compared with neutron doses obtained for RS and BC at the same distances $(13,20$ and $30 \mathrm{~cm}$ ) for a $5 \mathrm{yr}$ old phantom, which is presented in table 2. The obtained results are higher for closer distances, levelling out at a distance of about $30 \mathrm{~cm}$, which is the expected result due to the presence of a pre-absorber. Similar measurements were made by Hälg et al (2014) for proton PBS inside the anthropomorphic RANDO phantom. Considering the differences between these two experimental setups (phantom and target size, proton field parameters and set up) these results are in good agreement. 
Table 2. Neutron dose equivalent per target dose $H / D_{\mathrm{T}}\left(\mu \mathrm{Sv} \mathrm{Gy}^{-1}\right)$ as a function of distance from the isocentre for BD and PADC (UAB) for a 5 yr old phantom-comparison with literature data (Hälg et al 2014, Knežević et al 2018). The uncertainties for BD and PADC (UAB) detectors can be assessed using values for particular counters in table 2 in the supplementary data, while for results in Hälg et al (2014) the standard uncertainty of $28 \%$ was used.

\begin{tabular}{|c|c|c|c|c|c|c|c|}
\hline \multirow{3}{*}{$\begin{array}{l}\text { Distance from } \\
\text { isocentre }(\mathrm{cm})\end{array}$} & \multicolumn{7}{|c|}{ Neutron dose equivalent per target dose $H / D_{\mathrm{T}}\left(\mu \mathrm{Sv} \mathrm{Gy}^{-1}\right)$} \\
\hline & \multicolumn{3}{|c|}{ BD comparison } & \multicolumn{3}{|c|}{ PADC (UAB) comparison } & \multirow{2}{*}{$\begin{array}{l}\text { Track-etched detectors } \\
\text { Hälg et al (2014) }\end{array}$} \\
\hline & $\mathrm{RS}$ & $\mathrm{BC}$ & Knežević et al (2018) & RS & $\mathrm{BC}$ & Knežević et al (2018) & \\
\hline 13 & 195 & 133 & 130 & 280 & 226 & 130 & $\sim 500-600$ \\
\hline 20 & 57 & 46 & 30 & 107 & 48 & 50 & - \\
\hline 30 & 32 & 12 & - & 29 & 33 & 15 & $\sim 70$ \\
\hline
\end{tabular}

\section{Conclusions}

This paper reports the results of an experiment to determine the out-of-field doses during PBS irradiation of shallowly located tumours in children. Two treatment approaches with the use of additional proton energy pre-absorbers were considered. Radiation doses in the 5 and $10 \mathrm{yr}$ anthropomorphic phantoms and in the treatment room were determined for two types of pre-absorbers—an RS and a personalized 3D printed proton BC. The main purpose of this comparison was to check the impact of the latter on the scattered radiation doses compared with conventionally used RSs.

The results confirmed that the use of $\mathrm{BC}$ as the pre-absorber does not significantly increase and in many cases slightly decreases these out-of-field-doses while being highly useful during the treatment of shallow lesions. Considering and summarizing all the results and comparing the measured doses for RS and BC it is concluded that the use of personalized 3D printed proton compensator be can be safely used for paediatric patients.

\section{Acknowledgments}

This work was carried out within the European Radiation Dosimetry Group (EURADOS, WG9 Radiation Dosimetry in Radiotherapy).

The study was partially supported by the Horizon 2020 project INSPIRE Grant No. 730983. A W has been partly supported by the EU Project POWR.03.02.00-00-I004/16. I M-R acknowledges the financial support from the Spanish Ministry of Science, Innovation and Universities (fellowship RYC2018-024043-I).

The authors gratefully acknowledge the contribution and help from the staff of the Cyclotron Centre Bronowice, Krakow, Poland and also to Marijana Nodilo for the help in preparation and readout of RPL dosimeters.

\section{ORCID iDs}

A Wochnik (1) https://orcid.org/0000-0002-7848-1145

L Stolarczyk (1) https://orcid.org/0000-0002-6333-3350

S Domański (D) https://orcid.org/0000-0002-7847-2169

R Kopeć 이 https://orcid.org/0000-0002-0919-9859

M Kuć (i) https://orcid.org/0000-0003-2781-0743

N Mojżeszek (1) https://orcid.org/0000-0001-7528-1984

I Martínez-Rovira (1) https://orcid.org/0000-0002-2918-489X

M Á Caballero-Pacheco (1) https://orcid.org/0000-0002-2433-1125

J Swakoń i] https://orcid.org/0000-0001-9262-7326

S Trinkl (i) https://orcid.org/0000-0001-5352-5490

P Olko (i) https://orcid.org/0000-0001-5554-8178

\section{References}

Ambrožová I, Pachnerová Brabcová K, Shurshakov V A, Tolochek R V and Kodaira S 2019 Angular dependence of track-etch detectors HARZLAS TD-1 Radiat. Prot. Dosim. 186 219-23

Atchison F 1980 Spallation and fission in heavy metal nuclei under medium energy proton bombardement Proc. Meeting on Targets for Neutron Beam Spallation Sources Jul-Conf-34, (11-12 June) (Julich: Kernforschungsanlage Julich GmbH)

Baumer C, Janson M, Timmermann B and Wulff J 2018 Collimated proton pencil-beam scanning for superficial targets: impact of the order of range shifter and aperture Phys. Med. Biol. 638 
Bonfrate A et al 2016 Influence of beam incidence and irradiation parameters on stray neutron doses to healthy organs of pediatric patients treated for an intracranial tumor with passive scattering proton therapy Phys. Med. 32 590-9

Burgkhardt B, Fieg G, Klett A, Plewnia A and Siebert B R L 1997 The neutron fluence and H*(10) response of the new LB 6411 rem counter Radiat. Prot. Dosim. $70361-4$

Burleson S, Baker J, Hsia A T and Xu Z 2015 Use of 3D printers to create a patient-specific 3D bolus for external beam therapy J. Appl. Clin. Med. Phys. 16 166-78

Canters R A et al 2016 Clinical implementation of 3D printing in the construction of patient specific bolus for electron beam radiotherapy for non-melanoma skin cancer Radiat. Oncol. 121 148-53

Caresana M et al 2014 Intercomparison of radiation protection instrumentation in a pulsed neutron field Nucl. Instrum. Methods Phys. Res. A 737 203-13

Chadwick M B et al 1999 A consistent set of neutron kerma coefficients from thermal to $150 \mathrm{MeV}$ for biologically important materials Med. Phys. 26 974-91

Chadwick M B et al 2006 ENDF/B-VII.0: next generation evaluated nuclear data library for nuclear science and technology Nucl. Data Sheets 107 2931-3060

Domingo C, De-San-Pedro M, García-Fusté M J, Romero M T, Amgarou K and Fernández F 2013 Estimation of the response function of a PADC based neutron dosimeter in terms of fluence and $\mathrm{Hp}(10)$ Radiat. Meas. 50 82-86

Dresner L 1962 EVAP: a FORTRAN program for calculating the evaporation of various particles from excited compound nuclei Tech. Report ORNL-TM-196 (Oak Ridge, TN: Oak Ridge National Lab) (https://doi.org/10.2172/4638868)

Farah J et al 2015 Measurement of stray radiation within a scanning proton therapy facility: EURADOS WG9 intercomparison exercise of active dosimetry systems: characterization of stray neutrons in proton therapy Med. Phys. 42 2572-84

García M J, Amgarou K, Domingo C and Fernández F 2005 Neutron response study of two CR-39 personal dosemeters with air and Nylon converters Radiat. Meas. 40 607-11

García-Fusté M J, Devienne A, Romero-Expósito M, Caballero-Pacheco M A and Domingo C 2020 Calibration of neutron dosimeters for radiation protection use at the ALBA synchrotron experimental hall Radiat. Psychol. Chem. 171108749

Gillin M T et al 2010 Commissioning of the discrete spot scanning proton beam delivery system at the University of Texas M.D. Anderson Cancer Center, Proton Therapy Center, Houston Med. Phys. 37 154-63

Golnik N, Brede H J and Guldbakke S $1997 \mathrm{H}^{*}(10)$ response of the REM-2 recombination chamber in monoenergetic neutron fields Radiat. Prot. Dosim. 74 139-44

Golnik N, Gryzi ski M A, Kowalska M, Meronka K and Tulik P 2014 Characterisation of radiation field for irradiation of biologica samples at nuclear reactor-comparison of twin detector and recombination methods Radiat. Prot. Dosim. 161 196-200

Hälg R A, Besserer J, Boschung M, Mayer S, Lomax A J and Schneider U 2014 Measurements of the neutron dose equivalent for various radiation qualities, treatment machines and delivery techniques in radiation therapy Phys. Med. Biol. 59 2457-68

Jadrnícková I, Spurny F and Molokanov A G 2008 To the spectrometry of linear energy transfer in charged particle beams by means of track-etch detectors Phys. Part. Nuclei Lett. 5 531-7

Ju S G et al 2014 New technique for developing a proton range compensator with use of a 3-dimensional printer Int. J. Radiat. 88 453-8

Knežević Ž et al 2018 Comparison of response of passive dosimetry in scanning proton radiotherapy-a study using paediatric anthropomorphic phantoms Radiat. Prot. Dosim. 180 256-60

Knežević Ž, Stolarczyk L, Bessieres I, Bordy J M, Miljanić S and Olko P 2013 Photon dosimetry methods outside the target volume in radiation therapy: optically stimulated luminescence (OSL), thermoluminescence (TL) and radiophotoluminescence (RPL) dosimetry Radiat. Meas. 57 9-18

Kry S F et al 2017 AAPM TG 158: measurement and calculation of doses outside the treated volume from external-beam radiation therapy Med. Phys. 44 e391-e429

Kuznetsov V E, Solonin A N, Urzhumtsev O D, Schilling R and Tavitov A G 2018 Strength of PLA components fabricated with fused deposition technology using a desktop 3D printer as a function of geometrical parameters of the process Polymers 10313

Łukowiak M et al 2016 Utilization of a 3D printer to fabricate boluses used for electron therapy of skin lesions of the eye canthi J. Appl. Clin. Med. Phys. 18 76-81

Majer M et al 2017 Out-of-field dose measurements for 3D conformal and intensity modulated radiotherapy of a paediatric brain tumour Radiat. Prot. Dosim. 176 331-40

Mares V et al 2016 A comprehensive spectrometry study of a stray neutron radiation field in scanning proton therapy Phys. Med. Biol. 61 4127-40

Mares V, Sannikov A V and Schraube H 2002 Response functions of the Andersson-Braun and extended range rem counters for neutron energies from thermal to $10 \mathrm{GeV}$ Nucl. Instrum. Methods Phys. Res. A 476 341-6

Matysiak W, Yeung D, Slopsema R and Li Z 2016 Evaluation of the range shifter model for proton pencil-beam scanning for the Eclipse v.11 treatment planning system J. Appl. Clin. Med. Phys. 17 391-404

Mayer S, Golnik N, Kyllönen J E, Menzel H G and Otto T 2004 Dose equivalent measurements in a strongly pulsed high-energy radiation field Radiat. Prot. Dosim. $110759-62$

Michiels S et al 2018 Patient-specific bolus for range shifter air gap reduction in intensity-modulated proton therapy of head-and-neck cancer studied with Monte Carlo based plan optimization Radiother. Oncol. 128 161-6

Miljanić S, Bordy J-M, D’Errico F, Harrison R and Olko P 2014 Out-of-field dose measurements in radiotherapy-an overview of activity of EURADOS working group 9: radiation protection in medicine Radiat. Meas. 71 270-5

Mojżeszek N et al 2017 Measurement of stray neutron doses inside the treatment room from a proton pencil beam scanning system Phys. Med. 34 80-84

Murawski Ł, Maciak M, Gryzinski M A and Domanski S 2018 Investigation on radiation shielding properties of special concrete in neutron fields Radiat. Prot. Dosim. $180413-6$

Oh S A et al 2017 Fabrication of a patient-customized helmet with a three-dimensional printer for radiation therapy of scalp Prog. Med. Phys. 28100

Olsher R H et al 2000 WENDI: an improved neutron rem meter Health Phys. 79 170-81

Pachnerová Brabcová K, Ambrožová I, Kolísková Z and Malušek A 2013 Uncertainties in linear energy transfer spectra measured with track-etched detectors in space Nucl. Instrum. Methods A 713 5-10

Packer R J, Zhou T, Holmes E, Vezina G and Gajjar A 2013 Survival and secondary tumors in children with medulloblastoma receiving radiotherapy and adjuvant chemotherapy: results of Children's Oncology Group trial A9961 Neurol. Oncol. 15 97-103

Park S-Y, Choi C H, Park J M, Chun M, Han J H and Kim J 2016 A patient-specific polylactic acid bolus made by a 3D printer for breast cancer radiation therapy PloS One 11 e 0168063 
Pedroni E et al 2005 Experimental characterization and physical modelling of the dose distribution of scanned proton pencil beams Phys. Med. Biol. 50 541-61

Pelowitz D B 2011 MCNPX user's manual version 2.7.0 (Los Alamos, NM: Los Alamos National Laboratory) LA-CP-11-00438

Shen J et al 2015 Impact of range shifter material on proton pencil beam spot characteristics Med. Phys. 42 1335-40

Siebert B R L and Schuhmacher H 1995 Quality factors, ambient and personal dose equivalent for neutrons, based on the new ICRU stopping power data for protons and alpha particles Radiat. Prot. Dosim. 58 177-83

Stolarczyk L, Cywicka-Jakiel T, Horwacik T, Olko P, Swakon J and Waligorski M P R 2011 Evaluation of risk of secondary cancer occurrence after proton radiotherapy of ocular tumours Radiat. Meas. 46 1944-7

Stolarczyk L et al 2018 Dose distribution of secondary radiation in a water phantom for a proton pencil beam-EURADOS WG9 intercomparison exercise Phys. Med. Biol. 638

Tian X, Liu K, Hou Y, Cheng J and Zhang J 2018 The evolution of proton beam therapy: current and future status Mol. Clin. Oncol. 8 15-21

Titt $\mathrm{U}$ et al 2010 Adjustment of the lateral and longitudinal size of scanned proton beam spots using a pre-absorber to optimize penumbrae and delivery efficiency Phys. Med. Biol. 55 7097-106

Tulik P et al 2018 Investigation of secondary mixed radiation field around a medical linear accelerator Radiat. Prot. Dosim. $180252-5$

Valentin J 2003 Relative biological effectiveness (RBE), quality factor (Q), and radiation weighting factor (wR): ICRP Publication 92 Ann. ICRP 33 1-121

White D R, Booz J, Griffith R V, Spokas J J and Wilson I J 1989 Tissue substitutes in radiation dosimetry and measurement (report 44) J. ICRU os23 NP-NP

Zielczyński M, Golnik M and Gryziński M A 2008 Applications of recombination chambers in the dosimetry of high energy radiation fields Nukleonika 53 45-52

Zou W et al 2015 Potential of 3D printing technologies for fabrication of electron bolus and proton compensators J. Appl. Clin. Med. Phys. 16 90-98 